\title{
Antitumour effects of Yangzheng Xiaoji in human osteosarcoma: The pivotal role of focal adhesion kinase signalling
}

\author{
WEN G. JIANG ${ }^{1,2}$, LIN YE ${ }^{1,2}, \mathrm{KE} \mathrm{JI}^{1,2}$, FIONA RUGE ${ }^{1,2}$, YILING WU ${ }^{3}$, \\ YONG GAO ${ }^{3}$, JIAFU JI ${ }^{1,4}$ and MALCOLM D. MASON ${ }^{1,2}$ \\ ${ }^{1}$ Cardiff University-Peking University School of Oncology Joint Institute; ${ }^{2}$ Metastasis and Angiogenesis Research Group, \\ Institute of Cancer and Genetics, Cardiff University School of Medicine, Heath Park, Cardiff CF14 4XN, UK; \\ ${ }^{3}$ Yiling Medical Research Institute Tianshan Dajie, Shijiazhuang, Hebei; ${ }^{4}$ Department of Gastroenterological Oncology, \\ Key Laboratory of Carcinogenesis and Translational Research (Ministry of Education), \\ Peking University Cancer Hospital, Haidian, Beijing, P.R. China
}

Received April 17, 2013; Accepted May 22, 2013

DOI: 10.3892/or.2013.2586

\begin{abstract}
The present study examined, in vitro and in vivo, the potential antitumour effects of Yangzheng Xiaoji (YZXJ), a traditional Chinese medical formula used in cancer treatment, on osteosarcoma, a tumour type recently found to be sensitive to YZXJ. The human osteosarcoma cell line MG63 was used in cell-matrix adhesion and cell growth assays. The same cell line was used in an in vivo tumour model by establishing subcutaneous osteosarcoma xenografts. Oral and intraperitoneal routes were used to deliver the $Y Z X J$ extract. The effect of $Y Z X J$ on the activation of focal adhesion kinase (FAK) and paxillin was evaluated by immunofluorescence methods. It was found that $Y Z X J$ exhibited a significant inhibitory effect on cell-matrix adhesion as demonstrated by a cell-based assay and electric cell-substrate impedance sensing (ECIS) analysis. The effect was observed together with a reduction in phospho-FAK and phospho-paxillin in the cells when treated with YZXJ. In the in vivo tumour model, YZXJ was found to significantly inhibit the growth of osteosarcoma with a sustained effect observed when $Y Z X J$ was delivered intraperitoneally. $Y Z X J$ sensitized cells to the effect of FAK inhibitor in vitro and in vivo. It is concluded that Yangzheng Xiaoji plays a significant role in cell-matrix adhesion and tumour growth, likely by inhibiting the activation of the FAK pathway. The therapeutic role of Yangzheng Xiaoji in osteosarcoma warrants further investigation.
\end{abstract}

Correspondence to: Professor Wen G. Jiang, Metastasis and Angiogenesis Research Group, Institute of Cancer and Genetics, Cardiff University School of Medicine, Heath Park, Cardiff CF14 4XN, UK

E-mail: jiangw@cf.ac.uk

Key words: osteosarcoma, in vivo model, Yangzheng Xiaoji, cell adhesion, focal adhesion kinase, electric cell-substrate impedance sensing, paxillin, integrin

\section{Introduction}

Osteosarcoma, the most common type of primary bone cancer, occurs mostly in young age with more than $60 \%$ of cases noted in patients between 10 and 20 years of age and is most frequently present in lower long bones. The five-year survival of these patients is over $60 \%$ and varies with regard to age, gender, race and location of tumours (1-3). Treatments for osteosarcoma include surgery, chemotherapy (before and after surgery) and radiotherapy, although the latter is less effective when compared with surgery and chemotherapy. Other alternative therapies have been explored but are presently limited (4-6).

In a previous study, we reported that the traditional Chinese medical formula, Yangzheng Xiaoji, has a marked effect on angiogenesis The extract from Yangzheng Xiaoji, DME25, inhibited tubule formation in human endothelial cells (7). We demonstrated that this effect was through inhibition of focal adhesion kinase (FAK). Furthermore, we demonstrated that the same extract had a direct effect on a diverse range of human cancer cell lines including breast, prostate, gastric and colorectal cancer and other cancer cell lines (8), of which human osteosarcoma was found to be one of the most sensitive cell types.

Yangzheng Xiaoji has been shown, in clinical trials, to have anticancer activity in patients with certain solid tumours. For example, in patients with primary liver cancer, those who received conventional chemotherapy combined with Yangzheng Xiaoji $(\mathrm{n}=304)$ showed a significantly increased rate of disease remission (complete and partial remissions) compared with patients who received chemotherapy alone. Patients who received combinational therapy also had improved quality of life, based on the Karnofsky method $(9,10)$, and had improved immune functions.

There have been no reports on the antitumour effect of this formula in in vivo models, and no studies have been conducted in human osteosarcoma. In light of our recent study showing that osteosarcoma is sensitive to DME25, we carried out the present study in which we tested the biological impact 
of Yangzheng Xiaoji on osteosarcoma in vitro and tested the effect on tumour growth using an in vivo tumour model.

\section{Materials and methods}

Human osteosarcoma cell line, MG63 (ATCC-CRL1427 $7^{\mathrm{TM}}$ ) was purchased from ATCC (LGC, UK). The cells were maintained in Dubecco's modified Eagle's medium (DMEM) (Sigma-Aldrich, Poole, Dorset, UK) supplemented with penicillin, streptomycin and 10\% fetal calf serum (Sigma-Aldrich). The cells were incubated at $37^{\circ} \mathrm{C}$ in $5 \% \mathrm{CO}_{2}$ and $95 \%$ humidity. Matrigel (reconstituted basement membrane) was purchased from Collaborative Research Products (Bedford, MA, USA). A selective small inhibitor to FAK (FP573228) was from Tocris (Bristol, UK). The antibody to paxillin was from Transduction Laboratories, anti-integrin- $\beta 1$ was obtained from R\&D Europe, and anti-FAK and phospho-specific antibodies (anti-pFAK and anti-pPaxillin) were from Santa Cruz Biotechnology, Inc. (Santa Cruz, CA, USA).

Preparation of extract DME25 from Yangzheng Xiaoji for experimental use. The extract of Yangzheng Xiaoji (Yiling Pharmaceutical, Shijiazhuang, Hebei, China), DME25, was prepared as previously reported (11). The extract was standardised by quantifying the optical density of the preparation using a spectrophotometer at a wavelength of $405 \mathrm{~nm}$. A master preparation of the extract which exhibited an OD of 0.25 was stocked as the master stock and so named as DME25 for the experiments. Preparations from different batches of the formula were found to be consistent by way of chemical finger printing.

In vitro cell growth assay. MG63 cells were seeded into 96-well plates at a density of 3,000 cells/well. Triplicate plates were set up for overnight, 3- and 5-day incubation periods. Following sufficient incubation, the plates were removed from the incubator, fixed in $4 \%$ formaldehyde $(\mathrm{v} / \mathrm{v})$ and stained with $0.5 \%(w / v)$ crystal violet. The crystal violet stain was extracted using $10 \%$ acetic acid (v/v). The absorbance was determined using a spectrophotomer (Bio Tek ELx800; Bio Tek Instruments, Inc., Winooski, VT, USA).

Cell-matrix adhesion assay. The cell-motility adhesion assay was based on an established method (12). Briefly, solution containing $5 \mu \mathrm{g}$ Matrigel was added to each well of a 96-well tissue culture plate. This was allowed to air dry, after which the gel was rehydrated. MG63 cells $(4,000)$ were added to each well, in the presence of DME25, FAK inhibitor or the DME25/ FAK inhibitor combination or medium as control. After incubating at $37^{\circ} \mathrm{C}$ for $40 \mathrm{~min}$, the non-adherent cells were gently washed off using a multi-pipette. The adherent cells were fixed using a $4 \%$ formalin solution for $30 \mathrm{~min}$ and then stained with $0.5 \%$ crystal violet. The number of adherent cells were then counted under a microscope and expressed as the cell number per high power field.

Electric cell-substrate impedance sensing (ECIS)-based cellular adhesion assays. An ECIS-ZO instrument with a 96-well station (Applied Biophysics Inc., Troy, NJ, USA) was used for the cell adhesion assays $(13,14)$. Cell modelling was carried out using the ECIS RbA modelling software, supplied by the manufacturer. The 96W1E ECIS arrays were used. ECIS measures the interaction between cells and the substrate to which they are attached via gold-film electrodes placed on the surface of culture dishes. Following treatment of the array surface with a cysteine solution, the arrays were incubated with complete medium for $1 \mathrm{~h}$. The adhesion was tracked immediately after adding the cells into the arrays. Impedance and resistance of the cell layer were immediately recorded for a period of up to $4 \mathrm{~h}$. For signalling transduction inhibitor assays, the respective inhibitors were included in the assay wells. Adhesion was also modelled using the ECIS RbA cell modelling software as recently reported $(14,15)$.

In vivo development of osteosarcoma. Athymic female nude mice (nude CD-1), 4-6 weeks of age, were purchased from Charles River, UK, and maintained in filter-topped units. One hundred microliters of cell suspension ( 0.5 million MG63 cells in $0.5 \mathrm{mg} / \mathrm{ml}$ Matrigel) was subcutaneously injected at the scapula area. Each tumour group was divided into groups receiving, on alternate days: i) control buffer, ii) i.p. injection of DME25, iii) oral delivery (by way of gavage) of DME25, iv) FAK inhibitor or v) the combination of DME25 and the FAK inhibitor. Mice were weighed, and tumour sizes were measured twice weekly for 5 weeks. Mice with weight loss $>25 \%$ and tumour size $>1 \mathrm{~cm}$ in any dimension were sacrificed according to the UK Home Office and UKCCCR guideline. The volume of the tumour was determined using the formula: Tumour volume $=0.523 \mathrm{x}$ width ${ }^{2} \mathrm{x}$ length (16). At the conclusion of the experiment, animals were terminally sacrificed, primary tumours were dissected, weighed and frozen at $-80^{\circ} \mathrm{C}$. Part of the primary tumour was used for frozen sections for histological and immunohistological examinations.

Immunofluorescent staining of FAK and paxillin. Frozen sections of osteosarcoma tissues were cut at a thickness of $6 \mu \mathrm{m}$ using a cryostat (17). The sections were mounted on Super Frost Plus microscope slides, air-dried and then fixed in a mixture of 50\% acetone and 50\% methanol. The sections were then placed in Optimax wash buffer for 5-10 min to rehydrate. Sections were incubated for $20 \mathrm{~min}$ in $10 \%$ horse serum of blocking solution and probed with the primary antibody. Following extensive washings, sections were probed with specific antibodies to FAK and phospho-FAK (SC-1688 and SC-11766 respectively, from Santa Cruz Biotechnology, Inc.), paxillin and phospho-paxillin and integrin- $\beta 1$. Primary antibodies were made up in Tris buffer with $3 \%$ milk at a 1:100 concentration for $1 \mathrm{~h}$. The primary antibody was then completely removed by washing the cells 5 times in the same buffer. FITC-conjugated secondary antibodies (Sigma-Aldrich) were subsequently added to the cells, and the slides were incubated on a shaker platform in the dark for $1 \mathrm{~h}$. The slides were finally washed 3 times to remove the unbound secondary antibody, mounted with FluorSave ${ }^{\mathrm{TM}}$ (Calbiochem-Novabiochem Ltd., Nottingham, UK) and visualized under an Olympus BX51 fluorescence microscope at x100 objective magnification.

Similarly, immunofluorescent staining was also carried out on MG63 cells. Cells were placed in a chamber slide to adhere for $24 \mathrm{~h}$ before being treated with DME25 or FAK inhibitors. 

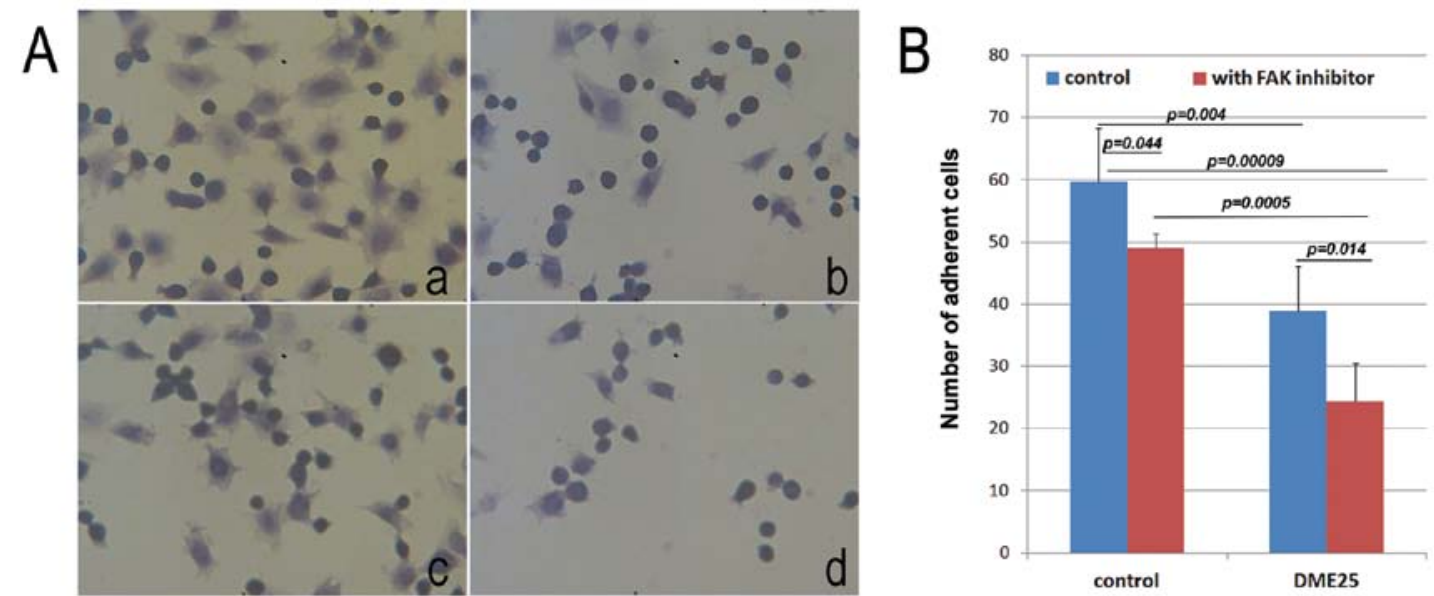

Figure 1. Effect of DME25 and the FAK inhibitor (100 nM) on the adhesiveness of MG63 osteosarcoma cells to a Matrigel-coated surface. (A) Representative images captured from the experimental settings: (a) control; (b) DME25 only (1:1,000); (c) FAK inhibitor only (100 nM) and (d) a combination of DME25 and FAK inhibitor. (B) Number of adherent cells per high power field.

A
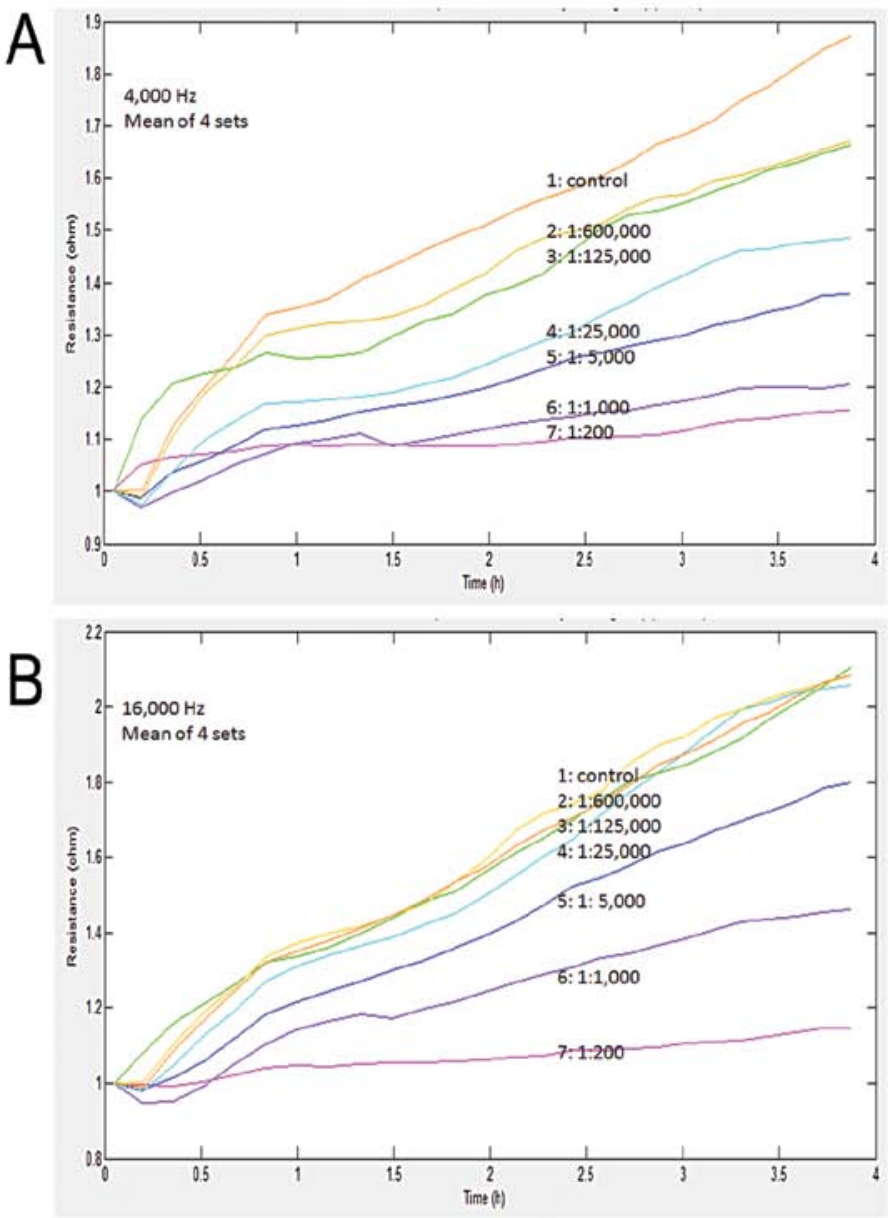

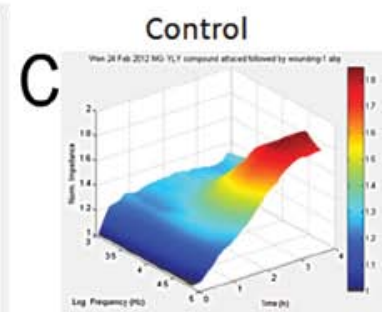

$1: 5000$
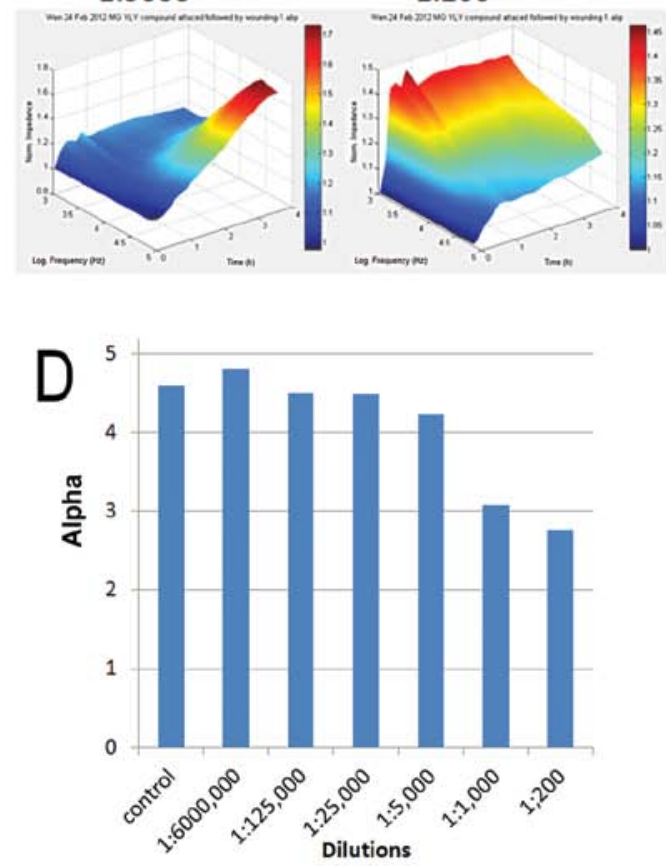

Figure 2. Effect of DME25 on the adhesiveness of osteosarcoma cells, using ECIS methods. DME25 was diluted from 1:200 to 1:600,000.

After the treatment, cells were first fixed with $4 \%$ formalin for $30 \mathrm{~min}$, before gently being permeabilized with Triton X-100 $(0.1 \%)$ for $5 \mathrm{~min}$. The rest of the procedure was similar to that used on tissues.

Statistical analysis was conducted using Sigma Plot (version 11).

\section{Results}

YZXJ has a direct effect on the adhesion of osteosarcoma cells. DME25 had a significant inhibitory effect on the adhesion of MG63 cells to a Matrigel-coated surface (Fig. 1A-a and B). The FAK inhibitor similarly inhib- 

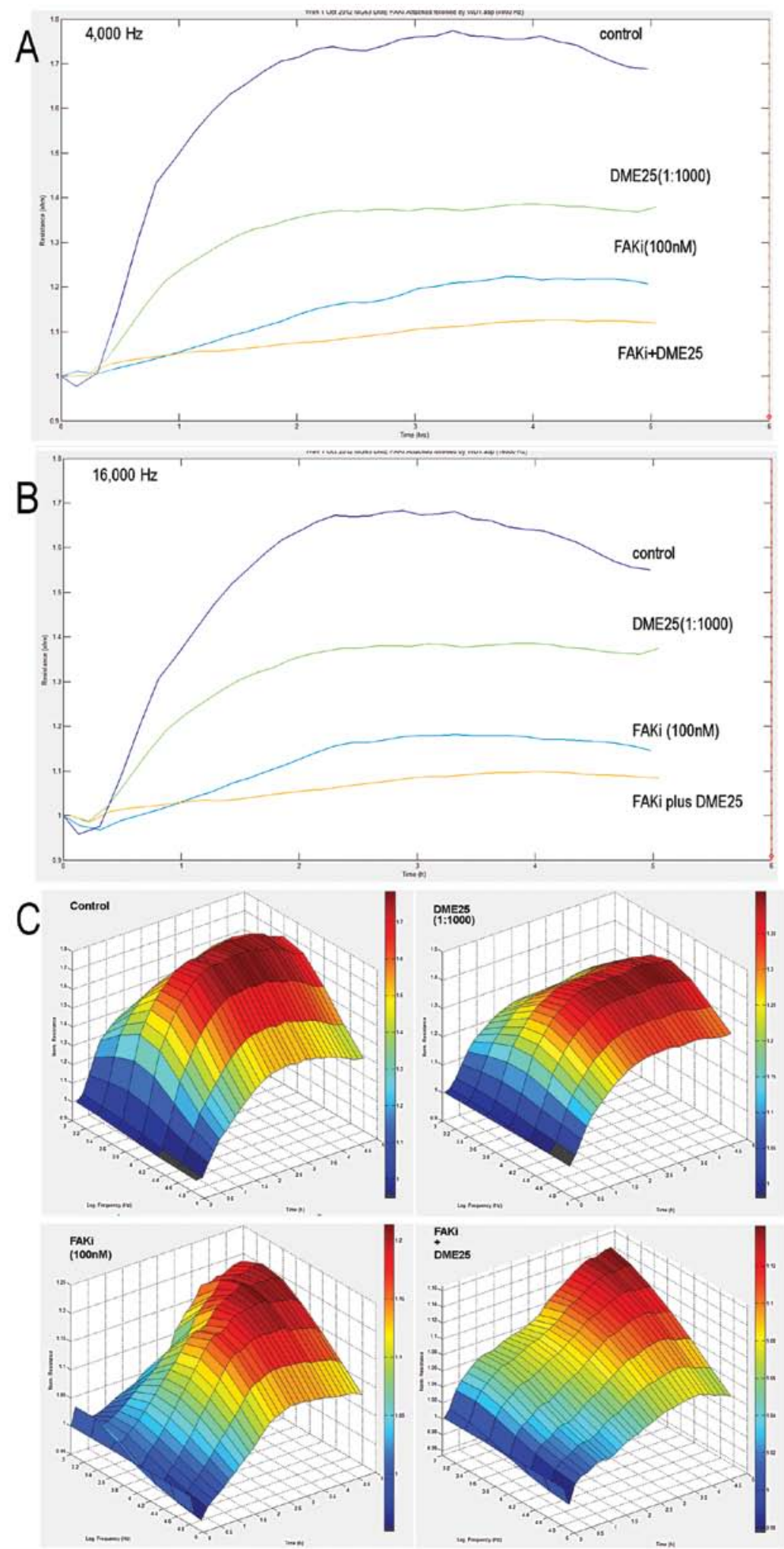

Figure 3. Effects of FAK inhibitor on cell adhesiveness as investigated by ECIS. (A and B) Traces of the cell response at 4,000 and 16,000 $\mathrm{Hz}$ (average of 4 data sets). (C) 3D demonstration of the cell response (average of 4 data sets). DME25 was used at 1:1,000 and the FAK inhibitor at $100 \mathrm{nM}$. Shown are the average of 8 traces. FAKi, FAK inhibitor.

ited the adhesion, although a weaker effect was noted when compared with that of DME25 at the concentration tested $(100 \mathrm{nM})$ (Fig. 1A-c and B). The combination of DME25 and the FAK inhibitor markedly potentiated the inhibitory effect on cell adhesiveness (Fig. 1A-d and B).
Using an automated ECIS method, we first demonstrated that DME25 had a marked effect on the adhesion of MG63 cells (Fig. 2). As shown in Fig. 2A, DME25 at a concentration as low as 1:125,000 dilution already demonstrated an inhibitory effect on cell adhesion, although a more 

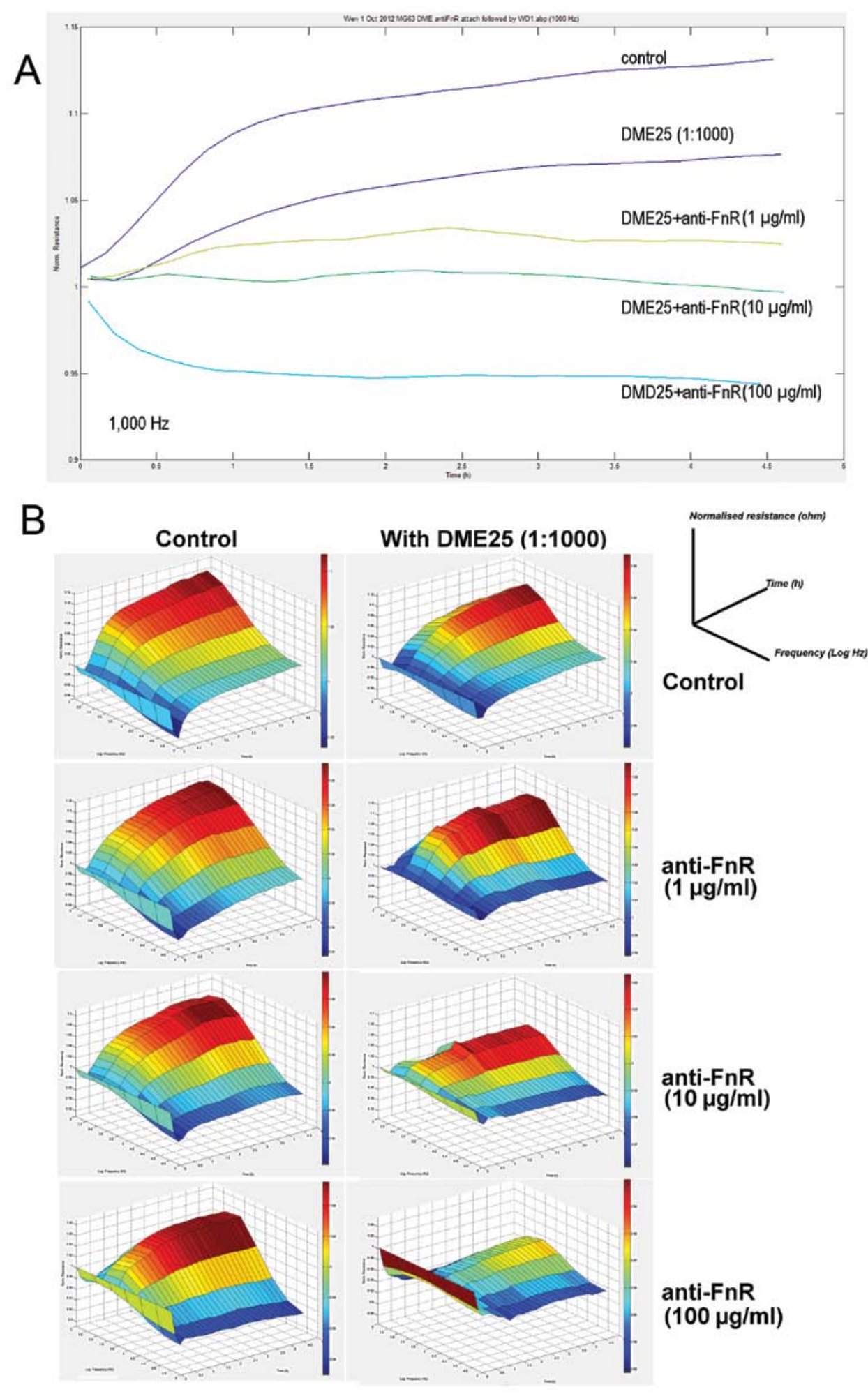

Figure 4. Anti-FnR (fibronectin receptor) inhibited cell-matrix adhesion, an effect further potentiated by DME25. (A) Example of traces (average of 4 data sets). (B) 3D demonstration (average of 4). Left column, cells treated with or without anti-FnR at different concentrations. Right column, cells treated with anti-FnR and DME25 at 1:1,000.

profound inhibitory effect was noted after treatment at a concentration higher than 1:25,000. A similar trend was noted at both low and high frequencies in the experimental settings (Fig. 2A and B). This inhibitory effect was noted across all of the frequencies tested as demonstrated in the 3D graph (Fig. 2C).
Inhibitory effects of the FAK inhibitor and anti-FnR on cell-matrix adhesion potentiated by YZXJ. As already shown in Fig. 1, the FAK inhibitor had an effect on cell-matrix adhesion. This was further demonstrated using the ECIS model (Fig. 3). We attempted to block the fibronectin receptor (FnR) by using a neutralising antibody. As shown in Fig. 4, the 

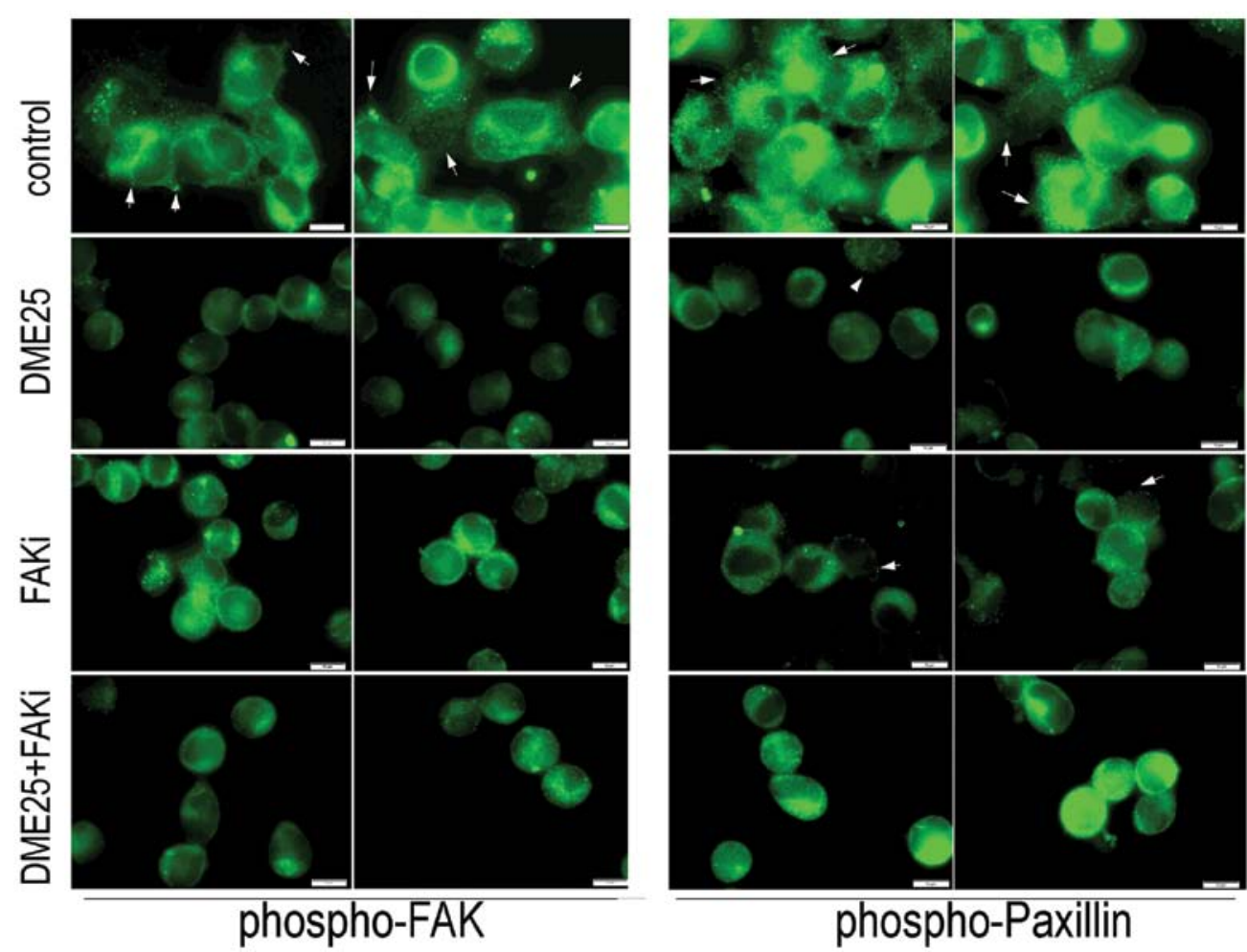

Figure 5. Staining of phosphorylated FAK (left panel) and paxillin (right panel) using an immunofluorescence method. Arrows indicate phosphorylated proteins at the focal adhesion areas of the cells. FAKi, FAK inhibitor.
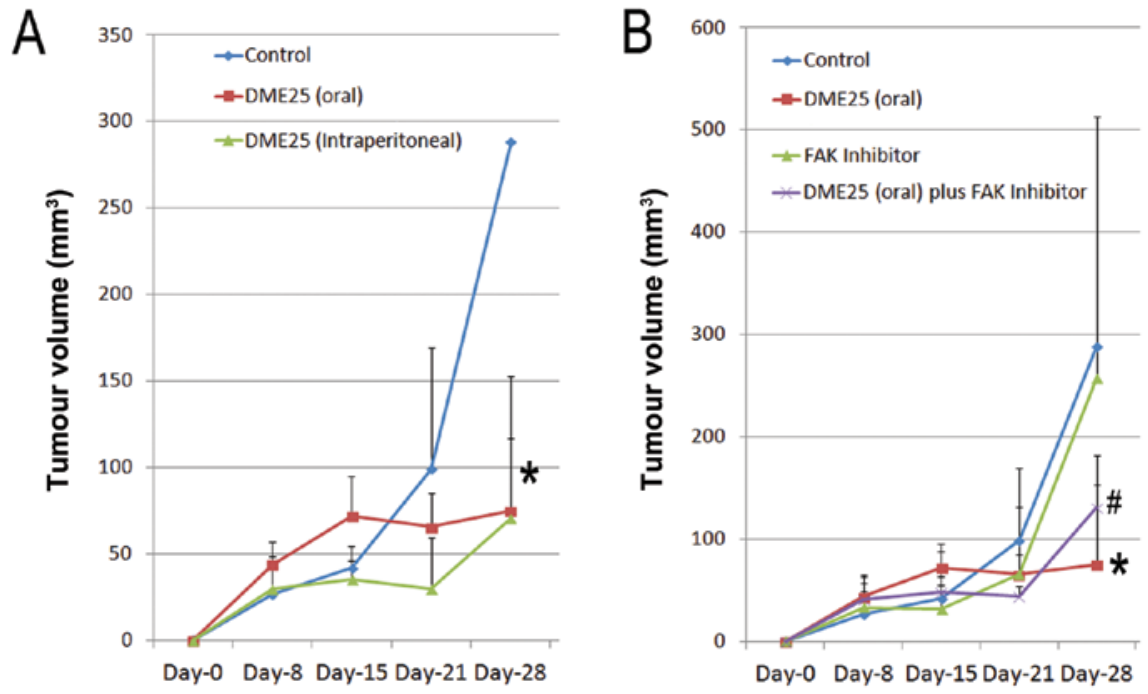

Figure 6. Effect of DME25 and the FAK inhibitor on in vivo tumour growth. Athymic nude mice were used. After tumours were established, mice received DME26 orally or intraperitoneally for up to 4 weeks. Shown are the volume of tumours $\left(\mathrm{mm}^{3}\right)$ following treatment with (A) DME25 and (B) FAKinhibitor in combination with DME25 at the specified times. "p $<0.05$ vs. control group; ${ }^{*} \mathrm{p}<0.05$ vs. FAK inhibitor only.

antibody reduced the cell adhesion. Again, the inhibition of cell adhesion by DME25 was further enhanced by anti-FnR.

YZXJ exhibits no significant effect on the growth of osteosarcoma cells. We recently reported that DME25 has an extremely small effect on the growth of a range of human cancer cell lines (8). In the present study, YZXJ at high concentrations did not show any toxic effect on cancer cells (data not shown).
Inhibition of the activation of FAK and paxillin by YZXJ in osteosarcoma cells. To investigate the effect of YZXJ on the activation of FAK and paxillin, we used an immunofluorescence method to visualize phosphorylated FAK and paxillin in MG63 cells. As shown in Fig. 5, control cells, which spread well over the matrix-coated surface, exhibited a high level of staining of phosphorylated FAK (Fig. 5, left panel, indicated by arrows) and phosphorylated paxillin (Fig. 5, right panel, 

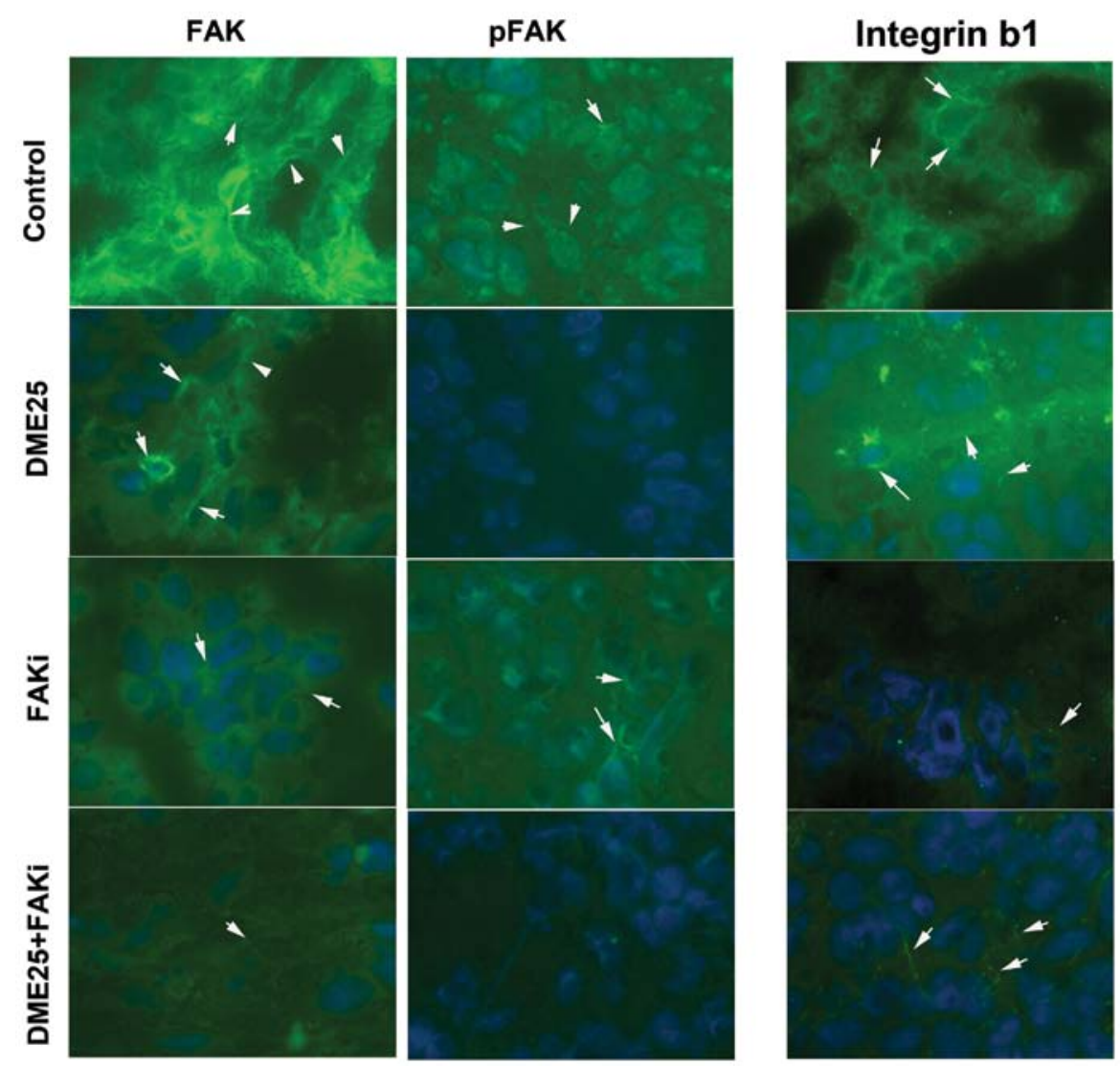

Figure 7. Staining of FAK (left panel), phospho-FAK (middle panel) and integrin (right panel) in osteosarcoma tumour sections. There was a marked reduction in pFAK in the treatment group in comparison with the control group. The changes in total FAK and integrin were less profound. FAKi, FAK inhibitor.

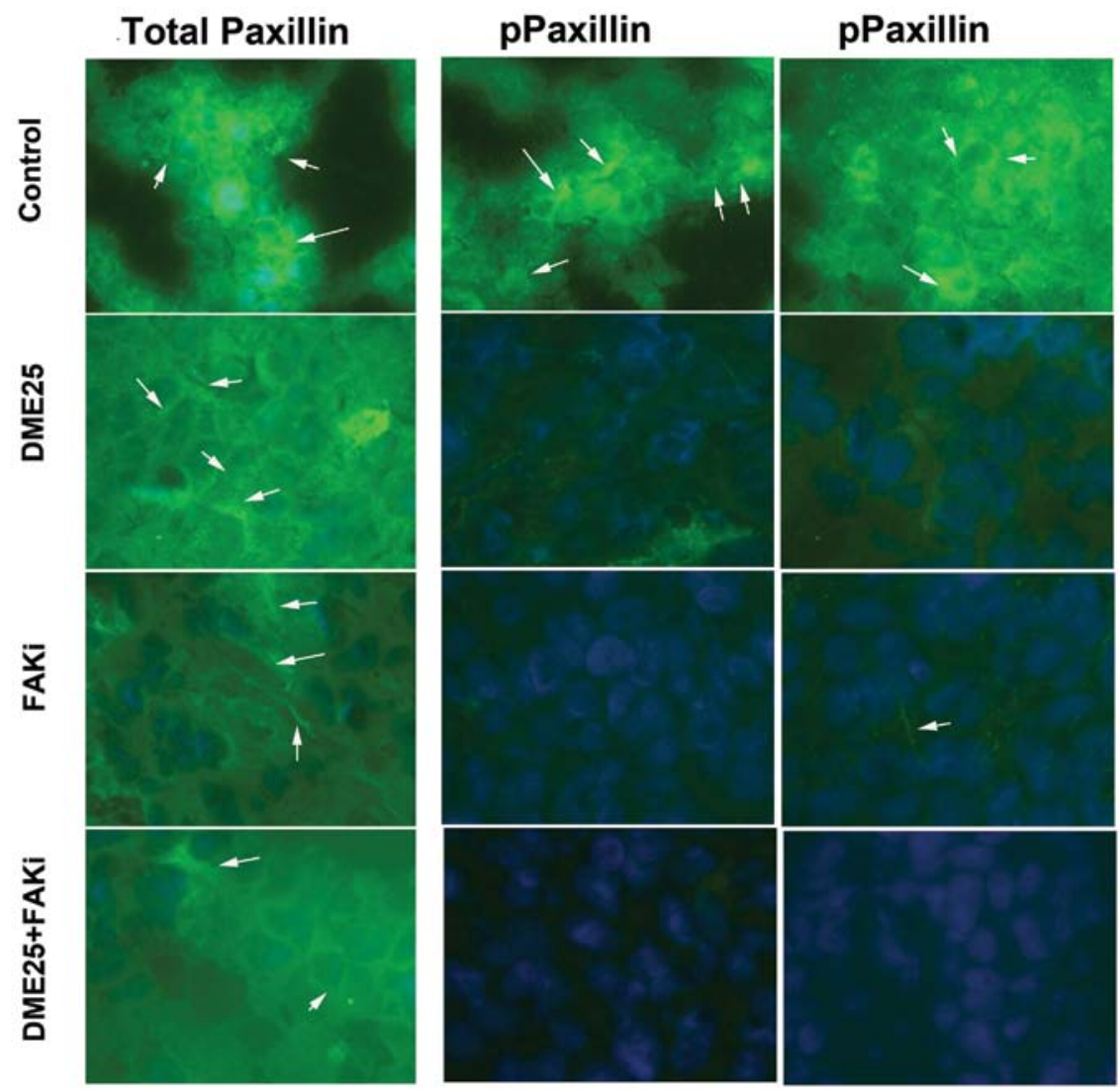

Figure 8. Staining of total paxillin (left panel) and phospho-paxillin (right panels). Marked reduction in pPaxillin was observed in the treated groups. FAKi, FAK inhibitor. 
indicated by arrows). The staining of both phosphorylated FAK and paxillin in the DME25-treated cells as well as in the FAK inhibitor-treated cells were markedly reduced, accompanied by morphological changes similar to that shown in Fig. 1. Likewise, the staining of both activated proteins was reduced in cells treated with the combination of DME25 and the FAK inhibitor.

YZXJ inhibits the growth of osteosarcoma in vivo. Using athymic nude mice, we first established subcutaneous osteosarcomas. The tumours became measurable after 1 week, when treatment began. As shown in Fig. 6A, delivery of DME25 via both the oral route and via intraperitoneal (i.p.) injection reduced the rate of growth, an effect which achieved statistical significance after 3 weeks. The i.p. injection resulted in sustained inhibition after 1 week whereas the oral route took longer than the i.p. route to reach a significant inhibitory effect. We also tested the effect of the FAK inhibitor and the combination of the FAK inhibitor and DME25. As shown in Fig. 6B, the FAK inhibitor at the concentration used (10 nM) showed little effect on tumour growth. However, the combination of DME25 and the FAK inhibitor exhibited a significant effect after 3 weeks. No obvious side-effects were observe throughout the study.

YZXJ and the activation of FAK and paxillin in the in vivo tumour models. We examined the staining pattern of FAK and paxillin by comparing the total proteins and phosphorylated forms using antibodies that recognized total FAK/paxillin or the phospho-specific proteins. Osteosarcoma tumour cells stained strongly for total FAK and also phospho-FAK (Fig. 7, indicated by arrows). Tumours from mice which received DME25, the FAK inhibitor and the DME25/FAK inhibitor combination showed a marked reduction in phospho-FAK staining, but to a lesser degree for the staining of total FAK. Similar to total FAK, a reduction in integrin staining was noted in tumours from mice that received treatment.

Fig. 8 shows a similar reduction in phospho-paxillin in the treatment group, except that the reduction was more profound that that noted with phospho-FAK (Fig. 7, left panel).

\section{Discussion}

In the present study, we demonstrated that DME25, previously shown to have an inhibitory effect on the adhesion and migration of human tumour cells, inhibited the cell-matrix adhesion of human osteosarcoma cells, possibly via an inhibitory effect on the activation of FAK and paxillin. This effect was noted together with a reduction in in vivo tumour growth and a reduction in the phosphorylation of FAK and paxillin in the osteosarcoma tumours.

The most significant finding of the present study was the antitumour effect of DME25, in vivo. Delivery of DME25 via both an oral route and an intraperitoneal route resulted in reduction in tumour growth. It is clear that the intraperitoneal route was more effective in the early days compared with the oral route. A method to monitor the absorption of DME25 in the body is yet to be developed. However, the different effects of oral and i.p. delivery may reflect the absorption and availability of DME25 to the tumours, as it is anticipated that an i.p. route may be more effective in regards to absorption. It is noteworthy that no side-effects were observed following the treatment regimes, suggesting that treatment under the present condition is safe.

Osteosarcoma cells markedly reduced their ability to adhere to matrix in the presence of DME25 at a broad range of concentrations. This was accompanied by a reduction in phospho-FAK and phospho-paxillin in the treated cells. This observation was similar to that noted in human endothelial cells with which DME25 reduced the phosphorylation of FAK (7). Together with a reduction in phospho-FAK and phospho-paxillin in osteosarcoma tumours, it is clear that the FAK pathway is a common target for DME25, both in endothelial and in tumour cells.

FAK plays an important role in cell-matrix adhesion, cellular migration and mediating intracellular and extracellular signals (18-20). Upon interaction between integrin and the extracellular matrix, the FAK pathway is activated which consequently activates downstream events including PI3K-Akt, Grb2-Erk, Crk-CAS. The finding of the present study that DME25 inhibits the activation of FAK and paxillin is in agreement with our previous study that DME25 inhibits the Akt pathway in other types of cancer cells including breast cancer and colorectal cancer cells (8); namely DME25 inhibits the early event in the FAK signalling chain of events.

FAK inhibitors are currently being tested in several clinical trials for treating patients with solid tumours (21-23). Here, we found that the FAK inhibitor only had a marginal effect on cell adhesion and tumour growth. This was primarily due to the choice of concentrations, namely below $100 \mathrm{nM}$, well below the recommended clinical dose. The concentrations were chosen in order to test whether DME25 may potentiate the effect of the inhibitor, which was indeed demonstrated both in the in vitro and in vivo models here. The pivotal role of the FAK pathway in the action of DME25 was further supported by the finding that blockage of the fibronectin receptor with neutralizing antibody also enhanced the effect of DME25. Together with the anti-angiogenic effect of DME25 (7), it can be argued that DME25 exerts antitumour effects by acting directly on tumour and endothelial cells, in which the FAK pathway is inhibited.

Osteosarcoma is an aggressive tumour mostly seen in young patients. Treatment includes surgery and chemotherapy. Surgery includes complete removal of the tumour when possible (24). Chemotherapy mostly involves a combination of multiple drugs, including adriamycin, ifosfamide, methotrexate and cisplatin. In a recent meta-analysis, it was indicated that a three-drug combination conferred a better survival benefit than a combination of less than three drugs $(25,26)$. Other types of therapies are also being explored including anti-angiogenesis therapy, interferons, and small-molecule inhibitors. The present study indicates that combination of DME25 and the FAK inhibitor may be a useful choice for treating patients with osteosarcoma. However, the present study was only based on one human osteosarcoma cell line, which happens to be highly sensitive to DME25. Thus, one should interpret the nature of tumour specificity with caution until more studies using other osteosarcoma cells are tested.

In conclusion, DME25, an extract from Yangzheng Xiaoji, has a profound inhibitory effect on the adhesion of osteo- 
sarcoma cells and on in vivo tumour growth. This is likely achieved by action on the focal adhesion kinase pathway in the cells.

\section{Acknowledgements}

The authors wish to thank Cancer Research Wales, the Albert Hung Foundation for supporting their study.

\section{References}

1. Mirabello L, Troisi RJ, and Savage SA: Osteosarcoma incidence and survival rates from 1973 to 2004: data from the Surveillance, Epidemiology, and End Results Program. Cancer 115: 1531-1543, 2009.

2. Dorfman HA and Czerniak B: Bone cancers. Cancer 75 (Suppl 1): S203-S210, 1995.

3. Hayden JB and Hoang BH: Osteosarcoma: basic science and clinical implications. Orthop Clin North Am 37: 1-7, 2006.

4. Wachtel $M$ and Schäfer BW: Targets for cancer therapy in childhood sarcomas. Cancer Treat Rev 36: 318-327, 2010.

5. Kubo T, Piperdi S, Rosenblum J, Antonescu CR, Chen W, Kim HS, Huvos AG, Sowers R, Meyers PA, Healey JH and Gorlick R: Platelet-derived growth factor receptor as a prognostic marker and a therapeutic target for imatinib mesylate therapy in osteosarcoma. Cancer 112: 2119-2129, 2008

6. Ferrari S and Palmerini E: Adjuvant and neoadjuvant combination chemotherapy for osteogenic sarcoma. Curr Opin Oncol 19: 341-346, 2007.

7. Jiang WG, Ye L, Ji K, Frewer N, Ji J and Mason MD: Inhibitory effects of Yangzheng Xiaoji on angiogenesis and the role of the focal adhesion kinase pathway. Int J Oncol 41: 1635-1642, 2012.

8. Ye L, Ji K, Frewer N, Ji J and Jiang WG: Impact of Yangzheng Xiaoji on the adhesion and migration of human cancer cells: the role of the AKT signalling pathway. Anticancer Res 32: 2537 $2543,2012$.

9. Zhang SY, Gu CH, Gao XD and Wu YL: A random, double-blinded and multicentre study of chemotherapy assisted Yangzheng Xiaoji capsule on treating primary hepatic carcinoma. Chin J Diffic Compl Case 8: 461-464, 2009.

10. Wang QL, Xuo CM, Wu XP, Li YX and Bi XJ: Treatment of atypical gastric dysplasia using Yangzheng Xiaoji. Chin J Diffic Compl Case 7: 38-39, 2009.

11. Ye Li, Ji K, Ji JF, Hargest R and Jiang WG: Application of electric cell-substrate impedance sensing in evaluation of traditional medicine on the cellular functions of gastric and colorectal cancer cells. Cancer Metastasis Biol Treat 17: 195-202, 2012

12. Jiang WG, Hiscox S, Hallett MB, Scott C, Horrobin DF and Puntis MC: Inhibition of hepatocyte growth factor-induced motility and in vitro invasion and motility of human colon cancer cells by gamma-linolenic acid. Br J Cancer 71: 744-752, 1995.

13. Giaever I and Keese CR: Micromotion of mammalian cells measured electrically. Proc Natl Acad Sci USA 88: 7896-7900, 1991.
14. Keese CR, Wegener J, Walker SR and Giaever I: Electrical wound-healing assay for cells in vitro. Proc Natl Acad Sci USA 101: 1554-1559, 2004.

15. Jiang WG, Martin TA, Lewis-Russell JM, Douglas-Jones A, Ye L and Mansel RE: Eplin-alpha expression in human breast cancer, the impact on cellular migration and clinical outcome. Mol Cancer 7: 71, 2008.

16. Davies G, Mason MD, Martin TA, Parr C, Watkins G, Lane J, Matsumoto K, Nakamura T and Jiang WG: The HGF/SF antagonist NK4 reverses fibroblast- and HGF-induced prostate tumor growth and angiogenesis in vivo. Int J Cancer 106: 348-354, 2003.

17. Ye L, Martin TA, Parr C, Harrison GM, Mansel RE and Jiang WG: Biphasic effects of 17- $\beta$-estradiol on expression of occludin and transendothelial resistance and paracellular permeability in human vascular endothelial cells. J Cell Physiol 196: 362-369, 2003.

18. Gilmore AP and Romer LH: Inhibition of focal adhesion kinase (FAK) signaling in focal adhesions decreases cell motility and proliferation. Mol Biol Cell 7: 1209-1224, 1996.

19. Chen JY, Tang YA, Huang SM, Juan HF, Wu LW, Sun YC, Wang SC, Wu KW, Balraj G, Chang TT, Li WS, Cheng HC and Wang YC: A novel sialyltransferase inhibitor suppresses FAK/paxillin signaling and cancer angiogenesis and metastasis pathways. Cancer Res 71: 473-483, 2011.

20. Stokes JB, Adair SJ, Slack-Davis JK, Walters DM, Tilghman RW, Hershey ED, Lowrey B, Thomas KS, Bouton AH, Hwang RF, Stelow EB, Parsons JT and Bauer TW: Inhibition of focal adhesion kinase by PF-562,271 inhibits the growth and metastasis of pancreatic cancer concomitant with altering the tumor microenvironment. Mol Cancer Ther 10: 2135-2145, 2011.

21. Halder J, Lin YG, Merritt WM, Spannuth WA, Nick AM, Honda T, Kamat AA, Han LY, Kim TJ, Lu C, Tari AM, Bornmann W, Fernandez A, Lopez-Berestein G and Sood AK: Therapeutic efficacy of a novel focal adhesion kinase inhibitor TAE226 in ovarian carcinoma. Cancer Res 67: 10976-10983, 2007.

22. Infante JR, Camidge DR, Mileshkin LR, Chen EX, Hicks RJ, Rischin D, Fingert H, Pierce KJ, Xu H, Roberts WG, Shreeve SM, Burris HA and Siu LL: Safety, pharmacokinetic, and pharmacodynamic phase I dose-escalation trial of PF-00562271, an inhibitor of focal adhesion kinase, in advanced solid tumors. J Clin Oncol 30: 1527-1533, 2012.

23. Soria JC, Plummer R, Ranson M, Gan H, Arkenau HT,Zalcman G, Blagden S, Evans TRJ, Peddareddigari V, Mazumdar J, Murray S, Gidson D, Fleming RA, Auger K and Millward M: Loss of the tumor suppressor merlin as a potential predictive biomarker of clinical activity for the oral, focal adhesion kinase (FAK) inhibitor $\mathrm{GSK}_{225698}$ in pts with recurrent mesothelioma. Eur J Cancer 48 (Suppl 6): S188, 2012.

24. Ritter J and Bielack SS: Osteosarcoma. Ann Oncol 21 (Suppl 7): vii320-vii325, 2010.

25. Anninga JK, Gelderblom H, Fiocco M, Kroep JR, Taminiau AH, Hogendoorn PC and Egeler RM: Chemotherapeutic adjuvant treatment for osteosarcoma: where do we stand? Eur J Cancer 47: 2431-2445, 2011

26. Jaffe N: Osteosarcoma: review of the past, impact on the future. The American experience. Cancer Treat Res 152: 239-262, 2009. 